\title{
recildunds
}

Revista Cientifica Mundo de la Investigación y el Conocimiento

Lissette Yesenia Rosero Ortega a ${ }^{\text {; }}$ Jorge Armando Rosero Aguirre ${ }^{\text {b; }}$ María

Stephania Limones Moncada ${ }^{c}$; Elvis Rodrigo Soledispa Cevallos ${ }^{\mathrm{d}}$

Obesidad y síndrome metabólico en pediatría

Obesity and metabolic syndrome in pediatrics

Revista Científica Mundo de la Investigación y el Conocimiento. Vol. 3 núm. 4., diciembre, ISSN: 2588-073X, 2019, pp. 456-478

DOI: $10.26820 /$ recimundo/3.(4).diciembre.2019.456-478

URL: http://recimundo.com/index.php/es/article/view/670

Código UNESCO: 3205 Medicina Interna

Tipo de Investigación: Artículo de Revisión

(C) RECIMUNDO; Editorial Saberes del Conocimiento, 2019

Recibido: 15/09/2019

Aceptado: 23/11/2019

Publicado: 30/12/2019

Correspondencia: karinaborjaa@gmail.com

a. Médico; Investigadora Independiente; Guayaquil, Ecuador; lissetterosero94@ gmail.com

b. Especialista En Imagenología; Doctor en Medicina y Cirugía; Investigador Independiente; Guayaquil, Ecuador; jorgrosero@hotmail.com

c. Médico; Investigadora Independiente; Guayaquil, Ecuador; sol-nia@ @otmail.com

d. Médico; Investigadora Independiente; Guayaquil, Ecuador; elvisrsc09@gmail.com 


\section{Obesidad y síndrome metabólico en pediatría}

Vol. 3, núm. 4., (2019)

Lissette Yesenia Rosero Ortega; Jorge Armando Rosero Aguirre; María Stephania Limones Moncada; Elvis Rodrigo Soledispa Cevallos

\section{RESUMEN}

La obesidad infantil puede acarrear complicaciones en cuanto al bienestar físico, social y emocional. Precisamente, el síndrome metabólico (SM) se encuentra dentro de las complicaciones físicas, y se trata de una de las complicaciones de la salud más comunes y trascendentes por la obesidad. En los últimos años, esta condición ha llamado poderosamente la atención en la especialidad de pediatría, debido a los niveles alarmantes en que se ubica la prevalencia de la obesidad infantil a nivel mundial, a su vez se vinculada con el incremento en la demanda de productos ultraprocesados en países de bajo y mediano desarrollo, como es el caso de la vulnerable región latinoamericana, a la que parece estar migrando este tipo de empresas. El objetivo de este trabajo es hacer referencia de las fuentes bibliográficas que de una manera práctica sustenten, no solamente la relación inequívoca entre la obesidad infantil y el síndrome metabólico en estos, sino además expliquen de qué se trata esté último. Los resultados obtenidos bastaron para exponer sobre algunas definiciones respecto a la obesidad y la obesidad infantil, su relación con el síndrome metabólico y los aspectos asociados a esta particular condición tales como causas, factores de riesgo, complicaciones, prevención, diagnóstico y tratamiento. De la investigación se concluye que, al tener presentes en todo momento la multiplicidad de factores de riesgo (exógenos y endógenos) que predisponen el síndrome metabólico, condición derivada principalmente por la obesidad, así como también pudiera ser debido a la dislipidemia, hipertensión arterial y resistencia insulínica, entonces es más probable que dicho síndrome sea abordado de manera adecuada.

Palabras Claves: Obesidad visceral; Enfermedad cardiovascular; Dislipidemias; Resistencia insulínica; Hiperglucemia. 


\section{Obesidad y síndrome metabólico en pediatría}

Vol. 3, núm. 4., (2019)

Lissette Yesenia Rosero Ortega; Jorge Armando Rosero Aguirre; María Stephania Limones Moncada; Elvis Rodrigo Soledispa Cevallos

\section{ABSTRACT}

Childhood obesity can lead to complications regarding physical, social and emotional wellbeing. Precisely, the metabolic syndrome (MS) is within the physical complications, and it is one of the most common and transcendent health complications due to obesity. In recent years, this condition has strongly called attention in the specialty of pediatrics, due to the alarming levels in which the prevalence of childhood obesity is located worldwide, in turn linked to the increase in demand for products ultraprocessed in countries of low and medium development, as is the case of the vulnerable Latin American region, to which this type of companies seems to be migrating. The objective of this work is to refer to the bibliographic sources that in a practical way support, not only the unequivocal relationship between childhood obesity and the metabolic syndrome in these, but also explain what the latter is about. The results obtained were enough to present some definitions regarding obesity and childhood obesity, its relationship with the metabolic syndrome and the aspects associated with this particular condition such as causes, risk factors, complications, prevention, diagnosis and treatment. The investigation concludes that, having in mind at all times the multiplicity of risk factors (exogenous and endogenous) that predispose the metabolic syndrome, a condition derived mainly from obesity, as well as could be due to dyslipidemia, arterial hypertension and insulin resistance, then it is more likely that such a syndrome is adequately addressed.

Keywords: Visceral obesity; Cardiovascular disease; Dyslipidemias; Insulin resistance; Hyperglycemia. 


\section{Obesidad y síndrome metabólico en pediatría}

Vol. 3, núm. 4., (2019)

Lissette Yesenia Rosero Ortega; Jorge Armando Rosero Aguirre; María Stephania Limones Moncada; Elvis Rodrigo Soledispa Cevallos

\section{Introducción.}

De acuerdo con Solís (2009), el fenómeno de la obesidad se encuentra vinculado con un cambio de la dieta a nivel global, ya que la tendencia se inclinó hacia el consumo de alimentos con un contenido calórico muy elevado; es decir, altas en carbohidratos y grasas y reducidas en vitaminas, minerales y micronutrientes, y al mismo tiempo con una disminución en la actividad física en razón del incremento de trabajos de naturaleza sedentaria, cambios en la forma de traslado o transporte y aumento de la urbanización. (Pág. 87).

En el mismo orden de ideas, Ordóñez \& Pérez (2016) refieren que la obesidad infantil es uno de los problemas que a nivel mundial viene gradualmente perturbando tanto en países de bajos ingresos como también en aquellos de medianos ingresos, incidiendo más aún en la población que reside en el medio urbano. Es uno de los problemas de salud pública más comprometidos del presente siglo y su prevalencia esta aumentado a un ritmo inquietante. Así mismo aseguran que "Se trata de un fenómeno multifactorial en el que intervienen desde factores personales como la ingesta de alimentos inadecuada en cantidad y calidad, sedentarismo, hasta los disruptores endocrinos, pasando por factores familiares, la condición socioeconómica, la herencia o la epigenética." (pág. 4).

En 2016 la OMS calculó que, a nivel mundial, más de 41 millones de niños menores de cinco años eran obesos o con sobrepeso y casi la mitad de estos vivían en Asia y una cuarta parte vivían en África.

El número de niños y adolescentes de edades comprendidas entre los cinco y los 19 años que presentan obesidad se ha multiplicado por 10 en el mundo en los cuatro últimos decenios. 


\section{Obesidad y síndrome metabólico en pediatría}

Vol. 3, núm. 4., (2019)

Lissette Yesenia Rosero Ortega; Jorge Armando Rosero Aguirre; María Stephania Limones

Moncada; Elvis Rodrigo Soledispa Cevallos

Las conclusiones de un nuevo estudio dirigido por el Imperial College de Londres y la Organización Mundial de la Salud (OMS) indican que, si se mantienen las tendencias actuales, en 2022 habrá más población infantil y adolescente con obesidad que con insuficiencia ponderal moderada o grave. (OMS, 2017).

Con estos datos iniciales no debe ser difícil comprender la idea que hasta no hace mucho dejaba clara Perea et al. (2014), ya que reconociendo los destacados adelantos en cuanto a la anatomía micro y macroscópica, la fisiología del tejido adiposo, las expresiones clínicas sistémicas derivadas de la obesidad y las estrategias de su diagnóstico y tratamiento, se atrevió a decir entonces que la obesidad puede ser asumida como "una enfermedad sistémica causante de múltiples complicaciones de fácil diagnóstico, de tratamiento frecuentemente complejo y con pocos resultados" (Pág. 317).

Instituciones como Mayo Clinic son enfáticas al afirmar que la obesidad infantil puede acarrear complicaciones en cuanto al bienestar físico, social y emocional. El síndrome metabólico se encuentra precisamente dentro de las complicaciones físicas, y al respecto detallan:

El síndrome metabólico es un grupo de trastornos que se presentan al mismo tiempo y aumentan el riesgo de enfermedad cardíaca, accidente cerebrovascular y diabetes tipo 2. Estos trastornos incluyen aumento de la presión arterial, niveles altos de azúcar en sangre, exceso de grasa corporal alrededor de la cintura y niveles anormales de colesterol o triglicéridos. (Mayo Clinic, 2019). 


\section{Obesidad y síndrome metabólico en pediatría}

Vol. 3, núm. 4., (2019)

Lissette Yesenia Rosero Ortega; Jorge Armando Rosero Aguirre; María Stephania Limones Moncada; Elvis Rodrigo Soledispa Cevallos

Cornejo \& Llanas (2013) en su obra han dejado claro que, es la población estudiada y la apreciación de los criterios diagnósticos lo que determina la variabilidad de la prevalencia del síndrome metabólico en pediatría, aunque obviamente también influye en su volumen la epidemia mundial de sobrepeso y obesidad infantil. Así mismo, estos tratadistas refieren múltiples datos relativos a la magnitud de esta complicación obtenidos de varios estudios previos en distintas regiones del mundo, por lo que en aras del propio contexto geográfico y la simplificación de la información que se procura aportar, se ha considerado rescatar lo relativo a las a aquellas cifras obtenidas en investigaciones hechas en las proximidades de la región latinoamericana, tal es el caso de los estudios adelantados en Brasil, Chile y Bolivia, al respecto refirieron:

En el estudio de escolares de Maracai, Brasil, la prevalencia del síndrome es de 3.6\% en general, pero cuando se analiza por condición de peso, ésta es de $0.3 \%$ para los de peso normal, de $10.7 \%$ para los de sobrepeso y de $34.5 \%$ para los de obesidad. [...] En los estudios de Chile, Burrows y su grupo refirieron una prevalencia de $4 \%$ en niños con sobrepeso y de $30 \%$ en niños con obesidad. [...] Caceres y colaboradores reportaron en niños de Bolivia, específicamente hispanos con sobrepeso, 32.2\% con síndrome metabólico. (Pág. 91).

Sin embargo, los autores no dejan de lado mencionar que:

Hasta el 2009, en una revisión sistemática de la literatura en ocho estudios, refieren una prevalencia que va de 4.2 a $15.4 \%$ con base en los criterios de NCEPATPIII y de 4.5 a $38.7 \%$ según los criterios de la OMS y el componente con mayor frecuencia encontrado fue la hipertrigliceridemia. (Pág. 91). 


\section{Obesidad y síndrome metabólico en pediatría}

Vol. 3, núm. 4., (2019)

Lissette Yesenia Rosero Ortega; Jorge Armando Rosero Aguirre; María Stephania Limones Moncada; Elvis Rodrigo Soledispa Cevallos

El objetivo de este trabajo es hacer referencia de las fuentes bibliográficas que de una manera práctica sustenten, no solamente la relación inequívoca entre la obesidad infantil y el síndrome metabólico en estos, sino además expliquen de qué se trata este último, por eso, a continuación se inicia presentando algunas definiciones de obesidad y acepciones sobre obesidad infantil, para luego enfatizar sobre los aspectos demostrativos del síndrome metabólico en pediatría, tales como su origen, factores de riesgo, complicaciones, prevención, diagnóstico y tratamiento.

\section{Materiales y Métodos.}

Las herramientas y materiales que fundamentalmente sirvieron para el desarrollo de este trabajo investigativo fueron: computadores personales con conexión a internet y cierta cantidad de contenidos bibliográficos científico académico, consultados tanto de manera física en la biblioteca universitaria y otras privadas, como también virtual, mediante la búsqueda de literatura de la misma índole en algunas bases de datos.

Mediante estos dos primeros procesos se ubicó y seleccionó un determinado cúmulo de información relativa a la obesidad y el síndrome metabólico en pediatría. Seguidamente, ese material fue leído, interpretado, analizado tanto individual como colectivamente en algunos casos, de manera tal que se discutiera de manera conjunta a fines de unificar criterios.

El diseño de la investigación se delimita al documental, de tipo no experimental, a nivel descriptivo, de corte transeccional y bajo una metodología de revisión, por lo que, en síntesis, el objetivo general del mismo se enfoca en la generación de un nuevo material bibliográfico en el que se recogen las ideas recientes de distintos autores en cuanto a la temática antes mencionada. 


\section{Obesidad y síndrome metabólico en pediatría}

Vol. 3, núm. 4., (2019)

Lissette Yesenia Rosero Ortega; Jorge Armando Rosero Aguirre; María Stephania Limones Moncada; Elvis Rodrigo Soledispa Cevallos

Algunas de las referidas bases de datos que fueron consultadas son: Biblioteca Virtual de la Salud (BVS), PubMed, Medline Plus, SciELO, Cochrane, Mayo Clinic, LILACS, entre otras.

Uno de los procesos mencionados se trató de la búsqueda en las mencionadas bases de datos, usando las expresiones "Obesidad pediátrica", "síndrome metabólico en pediatría" y "obesidad y síndrome metabólico en pediatría", y filtros tales como: idiomas, español e inglés; periodo de publicación, entre los años 2009 y 2019, ambos inclusive, salvo algunas excepciones; tipo de acceso, completo y abierto; área, salud, medicina, humanos, niños, pediatría; tipo de bibliografía, artículos científicos, revisiones sistemáticas, guías clínicas, e-books, ensayos clínicos, estudios de cohorte, estudios de casos, consensos, protocolos, tesis de grado, posgrado y doctorado, noticias científicas, boletines y/o folletos de instituciones oficiales o privadas de reconocida trayectoria en el área de la salud, medicina o científico académica, y demás, monografías y otros documentos que, a criterio del equipo, mostraran información de interés en base a la observación de la evidencia científica referida en sus contenidos. Este proceso arrojó un promedio de entre 3 y 42 resultado alusivos a enlaces de fuentes.

El otro proceso respondió a la selección y clasificación de la literatura científico académica físicamente disponible, teniendo en consideración criterios iguales o semejantes a los mencionados en el proceso antes descrito, siendo entonces a partir de allí que el equipo investigador le da la correspondiente lectura y análisis crítico de todo el contenido definitivamente seleccionado el cuál fue tratado como evidencia, siendo así que en consecuencia de manera consensuada se alcanza el fundamento de las ideas y planteamientos aquí expresados. 


\section{Obesidad y síndrome metabólico en pediatría}

Vol. 3, núm. 4., (2019)

Lissette Yesenia Rosero Ortega; Jorge Armando Rosero Aguirre; María Stephania Limones Moncada; Elvis Rodrigo Soledispa Cevallos

\section{Resultados.}

Con base en un reciente aporte de Castagnari (2019) para el Fondo de las Naciones Unidas para la Infancia (UNICEF), se comprende y en mayor medida, de allí puede asumirse que la problemática asociada al tema de la obesidad en pediatría bien puede estar contextualizado en el marco de la siguiente exposición:

La malnutrición, en todas sus formas, es la principal causa de problemas de salud a nivel global. La obesidad, en particular, ha crecido de manera alarmante, poniendo en riesgo muchos de los avances en salud que han logrado incrementar la expectativa de vida a nivel mundial, por lo que es urgente abordar la situación como un problema prioritario de salud pública. Se ha observado que la demanda de productos ultra procesados disminuye en países de altos ingresos, pero va en aumento en regiones más vulnerables como América Latina. Se ha verificado una relación inversamente proporcional entre el PBI de los países y el consumo de esos productos, que explica por qué las grandes compañías productoras están virando sus esfuerzos y su aparato de mercadeo hacia estas regiones. Estas tendencias, entonces, resaltan la necesidad de tomar acciones concretas en los mercados emergentes, más atractivos para las productoras de alimentos de bajo valor nutricional y con poblaciones más vulnerables. Aunque América Latina es una de las regiones del mundo que más avances ha logrado en la lucha contra la desnutrición crónica, es también una de las zonas más afectadas por el aumento del sobrepeso y la obesidad, que alcanza al 7,3\% de los menores de 5 años (cifra que supera ampliamente la media mundial del 5,6\%) (Castagnari, 2019). 


\section{Obesidad y síndrome metabólico en pediatría}

Vol. 3, núm. 4., (2019)

Lissette Yesenia Rosero Ortega; Jorge Armando Rosero Aguirre; María Stephania Limones Moncada; Elvis Rodrigo Soledispa Cevallos

La obesidad es una enfermedad inflamatoria, sistémica, crónica y recurrente, caracterizada por el exceso de grasa corporal y un sinnúmero de complicaciones en todo el organismo. Está directamente relacionada con el desarrollo y evolución de enfermedades como diabetes mellitus tipo 2, enfermedad cardiovascular, algunos tipos de cáncer, hipertensión arterial sistémica y otras. (Perea et al.; 2014; Pág. 317).

Particularmente, la posición del Consejo Europeo de Información sobre la Alimentación (EUFIC) sobre la obesidad en general y la obesidad infantil expresa que:

En términos simples, la obesidad se puede definir como una condición de acumulación excesiva o anormal de grasa en el tejido adiposo (graso) del cuerpo que conlleva riesgos e implicaciones para la salud. El sobrepeso y la obesidad se determinan a menudo a través del índice de masa corporal (IMC), que se calcula dividiendo el peso (en kilogramos) por la altura (en metros) al cuadrado $(\mathrm{kg} / \mathrm{m} 2)$. [...] En el caso de los niños y las niñas, los límites del IMC para el sobrepeso y la obesidad dependen de la edad y el género. Existen diferencias entre los patrones de crecimiento de los niños y las niñas que repercuten en la composición corporal y, por tanto, en el límite que indica si un niño o una niña presenta obesidad o sobrepeso. [...] La obesidad infantil no se debe a una única causa y el desequilibrio energético responde a la compleja interacción de varios factores (de riesgo), como el estilo de vida y el comportamiento, la genética, el entorno y la medicación, que se analizan más abajo. La dieta, el estado de salud y los comportamientos de estilo de vida de la madre también son indicadores importantes de la salud del nonato (EUFIC, 2017). 


\section{Obesidad y síndrome metabólico en pediatría}

Vol. 3, núm. 4., (2019)

Lissette Yesenia Rosero Ortega; Jorge Armando Rosero Aguirre; María Stephania Limones Moncada; Elvis Rodrigo Soledispa Cevallos

Hasta hace relativamente poco, la OMS publicó las nuevas directrices para profesionales de la salud en relación al problema de la obesidad infantil, al que por cierto se refiere como " una epidemia global que afecta tanto a los países ricos como a los menos desarrollados". Por cierto, dichas pautas coadyuvan en el alcance de los Objetivos de Desarrollo Sostenible, y además pertenecen al Plan de aplicación integral sobre la nutrición materna, infantil y del niño pequeño. La organización dejó claro que, de no adelantar acciones, un mayor riesgo de sufrir una variedad de enfermedades potencialmente peligrosas será padecido por los niños. Por ello ha insistido en que se proporcione la consejería "a los padres y cuidadores sobre nutrición y actividad física, incluyendo la promoción y apoyo para la lactancia materna" (OMS, 2017).

\section{Síndrome metabólico}

"El SM también se conoce como síndrome de resistencia insulínica (RI) o síndrome X. Es un conjunto de alteraciones que aumentan el riesgo de padecer enfermedades cardiovasculares y diabetes tipo 2." (García, 2015).

Güemes \& Muñoz (2015) lo definen como "un conjunto de factores de riesgo, que requieren la presencia de obesidad de predominio central, dislipidemia, hipertensión arterial y resistencia a la insulina, todos ellos, predictores de enfermedad cardiovascular y diabetes tipo 2 en el futuro." (Pág. 428).

Herrera (2015) ha indicado que:

Existen varias definiciones para el diagnóstico de este síndrome. Cook, en un intento de unificar criterios, propuso una definición pediátrica de este, modificando los 


\section{Obesidad y síndrome metabólico en pediatría}

Vol. 3, núm. 4., (2019)

Lissette Yesenia Rosero Ortega; Jorge Armando Rosero Aguirre; María Stephania Limones Moncada; Elvis Rodrigo Soledispa Cevallos

criterios establecidos por la ATP-III [Adult Treatment Panel III - Ver Tabla 1.], la cual ha sido muy utilizada en nuestro medio. Lo más relevante en esta clasificación es que ha puesto en evidencia que, si bien la prevalencia del síndrome es en general baja durante la infancia y adolescencia, no sucede lo mismo cuando los niños estudiados padecen de obesidad o sobrepeso. (Pág. 84-85).

En la literatura pediátrica se han realizado diversos intentos de caracterización del SM pediátrico con un significado similar al SM del adulto. Las dificultades para concretar una definición ampliamente aceptada de SM en la infancia y adolescencia son debidas en parte a la falta de valores normativos que puedan aplicarse en todo el mundo, a diferencias étnicas, valores normativos para las diferentes edades pediátricas, la ausencia de un rango de normalidad para la insulina en la infancia y la RI fisiológica de la pubertad. (Marta \& Joan, 2019, pág. 287).

\section{Causas}

García (2015) detalla que todavía no están definitivamente claras las interrelaciones entre los 5 elementos del SM tales como: obesidad abdominal, glucemia, presión arterial, triglicéridos y HDL-colesterol; y el rol de la RI, sin embargo, se cree que ésta última es principal responsable del síndrome ya que:

la RI se involucra en la patogénesis de la hipertensión arterial (aumenta la absorción renal de sodio y la actividad adrenérgica y antagoniza la acción del óxido nítrico) y de la dislipemia aterogénica (produce hipertrigliceridemia, descenso de colesterol-HDL y cambios cualitativos en las partículas de LDL-colesterol (unido a lipoproteínas de baja densidad), haciéndolas más pequeñas y densas, mucho más 


\section{Obesidad y síndrome metabólico en pediatría}

Vol. 3, núm. 4., (2019)

Lissette Yesenia Rosero Ortega; Jorge Armando Rosero Aguirre; María Stephania Limones Moncada; Elvis Rodrigo Soledispa Cevallos

aterogénicas). Hablamos de RI cuando la acción de esta hormona, la captación de glucosa por los tejidos periféricos y la supresión de la liberación de glucosa del hígado, se ve dificultada. En condiciones normales la RI se acompañará de una hiperinsulinemia por secreción aumentada compensadora para mantener la adecuada homeostasis de la glucosa. Antes de observar hiperinsulinemia basal, ya existe postprandrial. La captación celular de glucosa mediada por insulina varía entre una y diez veces en los sujetos sanos. Un 50\% aproximadamente de esta variabilidad puede atribuirse a factores exógenos (hábitos de vida) y el otro 50\% a características endógenas del individuo (Pág. 73-74).

Los factores exógenos a los se refiere el autor son: sobrepeso y la obesidad, ganancia ponderal rápida, inactividad física, dieta, estrés psicosocial, la falta de sueño y la depresión, y el uso crónico de algunos medicamentos; y entre los endógenos detalla: la raza, la pubertad, la predisposición genética a la RI y ciertos síndromes asociados con RI.

Mayo Clinic (2019) coincide igualmente con los criterios mencionados al inicio de la idea anterior, pero de manera más sencilla, explica que:

En condiciones normales, el sistema digestivo descompone los alimentos que ingieres y los transforma en azúcar. La insulina es una hormona generada por el páncreas que ayuda al ingreso del azúcar a las células para utilizarla como combustible. En las personas con resistencia a la insulina, las células no responden normalmente a la insulina y la glucosa no puede ingresar a las células con tanta facilidad. Como resultado, los niveles de glucemia aumentan incluso cuando tu cuerpo produce más insulina para intentar disminuir la glucemia. 


\section{Obesidad y síndrome metabólico en pediatría}

Vol. 3, núm. 4., (2019)

Lissette Yesenia Rosero Ortega; Jorge Armando Rosero Aguirre; María Stephania Limones Moncada; Elvis Rodrigo Soledispa Cevallos

Factores de riesgo

Los siguientes factores aumentan las posibilidades de tener síndrome metabólico:

- La edad. El riesgo de padecer síndrome metabólico aumenta con la edad.

- Origen étnico. En los Estados Unidos, los hispanos, en especial las mujeres hispanas, parecen tener el mayor riesgo de desarrollar síndrome metabólico.

- Obesidad. Tener sobrepeso, especialmente en el abdomen, aumenta el riesgo de síndrome metabólico.

- Diabetes. Es más probable que tengas síndrome metabólico si tuviste diabetes durante el embarazo (diabetes gestacional) o si tienes antecedentes familiares de diabetes tipo 2.

- Otras enfermedades. Tu riesgo de síndrome metabólico es más alto si alguna vez tuviste enfermedad del hígado graso no alcohólico, síndrome de ovario poliquístico o apnea del sueño. (Mayo Clinic, 2019).

De conformidad al consenso en la Guía ALAD "Diagnóstico, control, prevención y tratamiento del Síndrome Metabólico en Pediatría” (2016), otros factores son mencionados como predisponentes, entre los que figuran:

- Ser hijo de madre diabética o de madre con diabetes gestacional. 


\section{Obesidad y síndrome metabólico en pediatría}

Vol. 3, núm. 4., (2019)

Lissette Yesenia Rosero Ortega; Jorge Armando Rosero Aguirre; María Stephania Limones

Moncada; Elvis Rodrigo Soledispa Cevallos

- Tener bajo peso al nacimiento $(<2.5 \mathrm{~kg})$ así como el alto peso al nacimiento $(>4$ $\mathrm{kg})$.

- Recuperación ponderal a un ritmo muy rápido en los primeros meses de vida.

- Inicio de alimentación complementaria temprana (antes de los 6 meses de edad).

- Alimentación con leches industrializadas en los primeros 6 meses de edad.

- Ser hijo de padres con obesidad.

- No realizar ejercicio ni actividad física.

- Invertir más de 2 horas en actividades sedentarias (computadora, televisión, videojuegos, tareas escolares, etc.).

- Tener antecedentes familiares de DM2, HTA, infarto agudo de miocardio (IAM), enfermedad vascular cerebral (EVC).

- Comer más de dos veces por semana fuera de casa.

- Acostumbrar el consumo de bebidas que contienen azúcar como jugos, refrescos, etc.

- El bajo consumo de leche (menos de dos raciones al día). (ALAD, 2016)

\section{Complicaciones}

Tener síndrome metabólico puede aumentar el riesgo de desarrollar lo siguiente: 


\section{Obesidad y síndrome metabólico en pediatría}

Vol. 3, núm. 4., (2019)

Lissette Yesenia Rosero Ortega; Jorge Armando Rosero Aguirre; María Stephania Limones Moncada; Elvis Rodrigo Soledispa Cevallos

- Diabetes tipo 2. Si no realizas cambios en el estilo de vida para controlar tu exceso de peso, puedes desarrollar resistencia a la insulina, que puede causar un aumento en los niveles de azúcar en la sangre. Finalmente, la resistencia a la insulina puede provocar diabetes tipo 2 .

- Enfermedades del corazón y circulatorias. El nivel de colesterol alto y la hipertensión arterial pueden contribuir a la acumulación de plaquetas en las arterias. Estas plaquetas estrechan y endurecen las arterias, lo que puede producir un ataque cardíaco o un accidente cerebrovascular. (Mayo Clinic, 2019).

\section{Prevención}

Un compromiso de por vida con un estilo de vida saludable puede prevenir las afecciones que causan el síndrome metabólico. Un estilo de vida saludable incluye lo siguiente:

- Hacer actividad física al menos 30 minutos la mayoría de los días.

- Comer muchas verduras, frutas, proteínas magras y cereales integrales.

- Limitar la sal y las grasas saturadas en tu dieta.

- Mantener un peso saludable.

- No fumar. (Mayo Clinic, 2019) 


\section{Obesidad y síndrome metabólico en pediatría}

Vol. 3, núm. 4., (2019)

Lissette Yesenia Rosero Ortega; Jorge Armando Rosero Aguirre; María Stephania Limones Moncada; Elvis Rodrigo Soledispa Cevallos

\section{Diagnóstico}

Según las pautas del National Institutes of Health (Institutos Nacionales de Salud), se padece del síndrome metabólico cuando están presentes tres o más de los siguientes rasgos o se toman medicamentos para controlarlos:

- Circunferencia grande de cintura: una cintura que mide al menos 35 pulgadas (89 centímetros) en el caso de las mujeres y 40 pulgadas (102 centímetros) en el caso de los hombres.

- Niveles elevados de triglicéridos: 150 miligramos por decilitro (mg/dl) o 1.7 milimoles por litro (mmol/l) o niveles superiores de este tipo de grasa en sangre.

- Colesterol de lipoproteínas de alta densidad (HDL) o "bueno" reducido: menos de $40 \mathrm{mg} / \mathrm{dl}(1.04 \mathrm{mmol} / \mathrm{l})$ en los hombres o menos de $50 \mathrm{mg} / \mathrm{dl}(1.3 \mathrm{mmol} / \mathrm{l})$ en las mujeres.

- Hipertensión arterial: 130/85 milímetros de mercurio (mm Hg) o más.

- Glucemia en ayunas elevada: 100 mg/dl (5.6 mmol/l) o más. (Mayo Clinic, 2019)

Cornejo \& Llanas (2013) refieren que, desde 2007 la Federación Internacional de Diabetes (IDF) estableció los criterios diagnósticos del SM para la edad pediátrica (ver Tabla 1.), considerando la obesidad abdominal, la hipertrigliceridemia, la hipertensión y la disminución de C-HDL principales componentes de esta, así como también, factores adicionales tales como: el estado proinflamatorio, hiperuricemia, aumento de leptina y microalbuminuria. 


\section{Obesidad y síndrome metabólico en pediatría}

Vol. 3, núm. 4., (2019)

Lissette Yesenia Rosero Ortega; Jorge Armando Rosero Aguirre; María Stephania Limones Moncada; Elvis Rodrigo Soledispa Cevallos

Tabla 1. Criterios diagnósticos del síndrome metabólico en niños y adolescentes

\begin{tabular}{|c|c|}
\hline Criterios ATP III* & Criterios IDF** \\
\hline Púberes: & De 10 a 16 años: \\
\hline 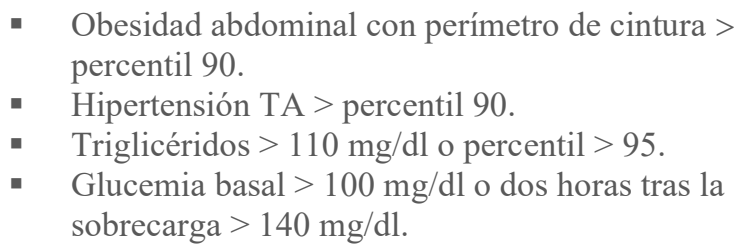 & 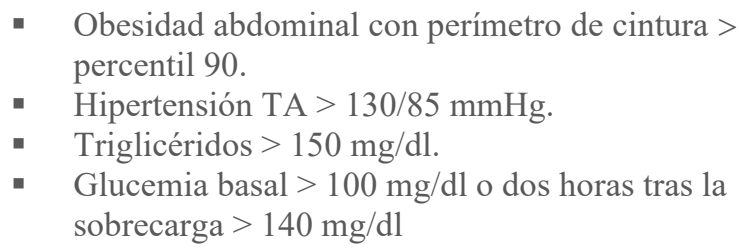 \\
\hline Prepúberes: & Menores de 10 años: \\
\hline - No hay criterios definidos & - No hay criterios definidos \\
\hline
\end{tabular}

Fuente: Recuperado de "Síndrome metabólico en niños y adolescentes " de Moreno, L.; Velasco, C. \& Matallana A. (2014), Gastrohnup. Vol. 16, № 2. Pág. 123

\section{Tratamiento}

Si los cambios radicales como dieta y ejercicio no son suficientes, tu doctor puede sugerir medicamentos para ayudar a controlar presión arterial, colesterol y nivel de azúcar en sangre.

- Hacer actividad física con regularidad.

- Pérdida de peso.

- Alimentarse sanamente.

- No fumar.

- Reducir o controlar del estrés. (Mayo Clinic, 2019). 


\section{Obesidad y síndrome metabólico en pediatría}

Vol. 3, núm. 4., (2019)

Lissette Yesenia Rosero Ortega; Jorge Armando Rosero Aguirre; María Stephania Limones Moncada; Elvis Rodrigo Soledispa Cevallos

En este sentido, Güemes \& Muñoz (2015) limitan su disertación al tratamiento nutricional; que abarca el ejercicio físico y aspectos psicológicos, y tratamiento farmacológico, haciendo énfasis en las recomendaciones para el tratamiento de Alteraciones de la tolerancia a la glucemia-Diabetes tipo 2, dislipemias e hipertensión arterial, aunque respecto a la obesidad, ofrece consultar aparte un tema que lo trata más ampliamente en otra entrega allí referida. En concreto se extrae:

Se deben realizar medidas preventivas, como cambios a estilos de vida saludables, incluyendo las medidas nutricionales y la actividad física, con el objetivo de mejorar la sensibilidad a la insulina y prevenir o corregir las alteraciones metabólicas y cardiovasculares asociadas. En cuanto a la composición de la dieta, se aconseja una dieta equilibrada, en la que el porcentaje de calorías aportadas por cada uno de los principios inmediatos sea: $50 \%$ en forma de hidratos de carbono, $30 \%$ de grasas y $20 \%$ de proteínas. Las proteínas deben ser de alto valor biológico. La grasa aportará ácidos grasos esenciales y vitaminas liposolubles, y favorecerá la sensación de saciedad. Asimismo, los alimentos con bajo índice glucémico ayudarán a controlar la sensibilidad a la insulina y las alteraciones lipídicas, así como asegurar un aporte extra de fibra que produce mayor sensación de saciedad y disminuye la ingesta energética. [...] El ejercicio físico regular puede mejorar la sensibilidad a la insulina en un $40 \%$ y disminuir la lipogénesis. Además, mejora los niveles de C-HDL y la función endotelial. Es útil para mantener la pérdida de peso y debe ser individualizado, realizando 30-60 minutos de ejercicio aeróbico diario. Las actividades sedentarias deben realizarse durante menos de 2 horas al día. [...] Se ha observado que los síntomas depresivos y el SM están unidos en ambas direcciones. Así, determinadas características psicológicas como la depresión y la hostilidad, pueden incrementar el riesgo para 


\section{Obesidad y síndrome metabólico en pediatría}

Vol. 3, núm. 4., (2019)

Lissette Yesenia Rosero Ortega; Jorge Armando Rosero Aguirre; María Stephania Limones Moncada; Elvis Rodrigo Soledispa Cevallos

el desarrollo de SM. [...] Los diferentes consensos y guías clínicas recomiendan el inicio del tratamiento farmacológico a partir de los 10 años de edad o en varones al inicio de la pubertad (Tanner II) y en mujeres tras la primera menstruación, después de un tratamiento dietético adecuado de seis meses a un año, siempre que los niveles de C-LDL sean superiores a $190 \mathrm{mg} / \mathrm{dl}$ o a $160 \mathrm{mg} / \mathrm{dl}$ y existan antecedentes familiares de enfermedad cardiovascular o el niño tenga dos o más factores de riesgo asociados. Entre los fármacos que podemos disponer en la infancia y la adolescencia están los siguientes; Resinas de intercambio iónico (...) Estas sustancias no tienen toxicidad sistémica, ya que no se absorben, por lo cual son potencialmente seguras en niños. [...] Ezetimibe: se localiza en las microvellosidades del intestino delgado e inhibe la absorción de colesterol, reduciendo su paso hacía el hígado. Posiblemente, por su mecanismo de acción y la casi ausencia de efectos adversos, va a ser el sustituto ideal de las resinas, mucho peor toleradas y menos eficaces. Sería el paso inicial junto a la dieta en el tratamiento de los hipercolesterolemias. Estatinas: disminuyen la síntesis endógena de colesterol mediante inhibición competitiva de la enzima HMG-CoA reductasa, enzima limitante en la biosíntesis del colesterol. [...] Fibratos: son sustancias químicas derivadas del ácido fíbrico (ácido clorofenoxiisobutírico). Actúan estimulando los receptores nucleares denominados "receptores activados de proliferación de los peroxisomas" (PPAR), en concreto los alfa. Dan lugar a un aumento del catabolismo de las partículas ricas en TG y a una disminución plasmática de las concentraciones de VLDL y triglicéridos. Producen un aumento de HDL-C y una disminución moderada del LDL-C. Los más utilizados son: bezafibrato, fenabibrato y gemfibrozil. (Pág. 432 434). 


\section{Obesidad y síndrome metabólico en pediatría}

Vol. 3, núm. 4., (2019)

Lissette Yesenia Rosero Ortega; Jorge Armando Rosero Aguirre; María Stephania Limones Moncada; Elvis Rodrigo Soledispa Cevallos

\section{Conclusiones.}

Queda más que claro que, primero que nada, la obesidad infantil todavía se considera una carga para la salud física, el bienestar mental e incluso las economías a nivel global, de allí que lo que hoy día es más trascendente es diagnosticarla, prevenirla y tratarla de manera oportuna.

A pesar de que existan considerables evidencias que sustentes el descenso de su prevalencia en países desarrollados, al parecer la tendencia es inversa en países de bajo y mediano desarrollo, como lo representan varios Estados de la región latinoamericana, por ello, es posible atreverse a decir que es igualmente importante que esta complejidad sea abordada de manera multidisciplinaria y en varios niveles en pro del mejoramiento de la salud pública.

Se recalca que, basados en la evidencia probabilística que sustenta la relación persistente entre la obesidad en la etapa adulta con la pediátrica (aparte de otros probables padecimientos de salud), se vuelve sumamente importante prevenir la obesidad en la infancia puesto que, en esa etapa, por una parte, son más sensibles a la influencia de los mensajes del entorno, y por la otra, se encuentran en medio de un proceso de establecimiento de hábitos que muy probablemente mantendrán en la adultez.

En síntesis, al tener presentes en todo momento la multiplicidad de factores de riesgo (exógenos y endógenos) que predisponen el síndrome metabólico, condición derivada principalmente por la obesidad, así como también pudiera ser debido a la dislipidemia, hipertensión arterial y resistencia insulínica, entonces es más probable que dicho síndrome sea abordado de manera adecuada. 


\section{Obesidad y síndrome metabólico en pediatría}

Vol. 3, núm. 4., (2019)

Lissette Yesenia Rosero Ortega; Jorge Armando Rosero Aguirre; María Stephania Limones Moncada; Elvis Rodrigo Soledispa Cevallos

\section{Bibliografía.}

ALAD. (2016). Guía ALAD "Diagnóstico, control, prevención y tratamiento del Síndrome Metabólico en Pediatría". Obtenido de ALAD-americalatina: http://www.aladamericalatina.org/wp-content/uploads/2016/10/SX-METABOLICO-EN-PEDIATRIA.pdf

Castagnari, V. (Abril de 2019). Obesidad: una cuestión de derechos de niños, niñas y adolescentes. Recomendaciones de políticas para su protección. Obtenido de UNICEF: https://www.unicef.org/argentina/media/4996/file/Obesidad,\%20una\%20cuesti\%C3\%B3 n\%20de\%20derechos.pdf

Cornejo, J., \& Llanas, J. (2013). Epidemiología del síndrome metabólico en pediatría. Evidencia Médica e Investigación en Salud, 6(3), 89-94.

EUFIC. (27 de Abril de 2017). Revisión de la obesidad infantil. Obtenido de EUFIC: https://www.eufic.org/es/healthy-living/article/childhood-obesity-review

García, E. (2015). Obesidad y síndrome metabólico en pediatría. En A. -A. Primaria (Ed.), $12^{\circ}$ Curso de Actualización Pediatría 2015. 3.0, págs. 71-84. Madrid: Asociación Española de Pediatría de Atención Primaria. Recuperado el 10 de 12 de 2019, de https://www.aepap.org/sites/default/files/cursoaepap2015p71-84.pdf

Güemes, M., \& Muñoz, M. (2015). Síndrome metabólico. Pediatría Integral, 9(6), 428-435.

Herrera, O. (Marzo de 2015). Síndrome metabólico en la infancia, evaluación dirigida a la atención primaria de salud. Revista Cubana de Pediatría, 87(1), 82-91.

Marta, M., \& Joan, B. (2019). Obesidad y síndrome metabólico. Protoc diagn ter pediatr, 1(1), 285-294.

Mayo Clinic. (20 de Junio de 2019). Síndrome metabólico. Obtenido de Mayo Clinic: https://www.mayoclinic.org/es-es/diseases-conditions/metabolic-syndrome/symptomscauses/syc-20351916

Moreno, L., Velasco, C., \& Matallana, A. (2014). Síndrome metabólico en niños y adolescentes. Gastrohnup, 16(2), 121-131.

OMS. (11 de Octubre de 2017). La obesidad entre los niños y los adolescentes se ha multiplicado por 10 en los cuatro últimos decenios. Obtenido de Organización Mundial de la Salud: https://www.who.int/end-childhood-obesity/es/

OMS. (04 de Octubre de 2017). La OMS lanza nuevas directrices para combatir la epidemia de obesidad infantil. Obtenido de Noticias ONU: https://news.un.org/es/story/2017/10/1387281 


\section{Obesidad y síndrome metabólico en pediatría}

Vol. 3, núm. 4., (2019)

Lissette Yesenia Rosero Ortega; Jorge Armando Rosero Aguirre; María Stephania Limones

Moncada; Elvis Rodrigo Soledispa Cevallos

Ordóñez, Á., \& Pérez, J. (Abril de 2016). Obesidad Infantil. Alimentación, actividad física y medio ambiente. (C. d. Sanidad, Ed.) Obtenido de SEAPA: https://www.seapaonline.org/UserFiles/File/Ayuda\%20en\%20consulta/2016/ObesidadInf antil\%20WEB.pdf

Perea, A., López, G., Padrón, M., Lara, A., Santamaría, C., Ynga, M., . . . . (2014). Evaluación, diagnóstico,tratamiento y oportunidades de prevención de la obesidad. Acta Pediátrica Mexicana, 35(4), 316-337.

Solís, J. (2009). Obesidad en la población pediátrica. Acta Pediátrica Costarricense, 21(2), 86 89.

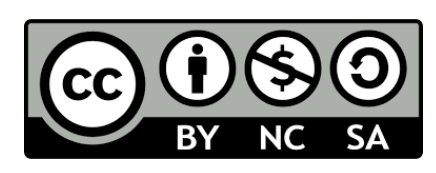

RECONOCIMIENTO-NOCOMERCIAL-COMPARTIRIGUAL

CC BY-NC-SA

ESTA LICENCIA PERMITE A OTROS ENTREMEZCLAR, AJUSTAR Y CONSTRUIR A PARTIR DE SU OBRA CON FINES NO COMERCIALES, SIEMPRE Y CUANDO LE RECONOZCAN LA AUTORÍA Y SUS NUEVAS CREACIONES ESTÉN BAJO UNA LICENCIA CON LOS MISMOS TÉRMINOS. 